Current Research in Psychology 1 (2): 102-107, 2010

ISSN 1949-0178

(C) 2010 Science Publications

\title{
Contrasting Three Popular Explanations for the Muller-Lyer Illusion
}

\author{
Michael R. Woloszyn \\ Thompson Rivers University, 900 McGill Road, Kamloops, BC Canada, V2C 5N3, Canada
}

\begin{abstract}
Problem statement: Using the method of adjustment, participants compared the line lengths of 'dumbbell' and 'spectacle' versions of Muller-Lyer (circles and ovals at the endpoints in place of arrowheads). Approach: Three popular competing explanations for the illusion (conflicting cues, misapplied size constancy scaling and confusion hypothesis) make differing predictions concerning the pattern of change in illusion strength when the bounding elements are varied. Results: PSEs were computed for circle and spectacle versions of Muller-Lyer, in which the end points were varied in size or orientation. A set of planned comparisons were carried out between the baseline versions of the illusion (with small endpoints) and the other configurations where the inner, outer, or both bounding elements were altered. Increasing the size of the inner bounding circles, as well as the inner and outer bounding spectacles, gave rise to significant increases in the illusion size (although the effect was not additive), however increasing the size of the outer bounding circles resulted in an unexpected significant decrease in illusion size not predicted by any model considered here (all differences significant at $\mathrm{p}<0.005$ ). It is possible that this unusual result is due to the bounding elements being perceived as separate from the line being bound. Conclusion/Recommendations: In summary, Confusion Hypothesis came closest to predicting the observed pattern of results however a complete explanation requires a combination of Misapplied Size Constancy Scaling, Confusion Hypothesis and the Ebbinghaus illusion.
\end{abstract}

Key words: Muller-Lyer illusion, constancy scaling, confusion hypothesis, spectacle stimuli, dumbbell stimuli, bounding circles, misapplied size, ebbinghaus illusion

\section{INTRODUCTION}

The Muller-Lyer illusion, in which two lines are made to appear to be different lengths by manipulating the bounding arrowheads at their endpoints (Fig. 1A), has received extensive study over the years and many candidate explanations for it have been proposed (Robinson, 1998). Three popular accounts, Misapplied Size Constance Scaling, Conflicting Cues and Confusion Hypothesis, form the focus of the study presented here.

According to Misapplied Size Constancy Scaling (Gregory, 1997) the illusion is due to the perceptual system misperceiving the lines as being different distances from the observer, which in turn, affect their relative perceived sizes, due to the bounding arrowheads being interpreted as conveying threedimensional information. Although there is some supportive evidence for this notion (Madden and Burt, 1981), critics point out that the size constancy scaling model offers no explanation for how, in the original Muller-Lyer configuration, the two lines are actually perceived to be different distances relative to one another, as opposed to relative to their own inducing elements (Rock, 1975). Moreover, many studies have challenged this central assumption of distance inferences in Misapplied Size Constancy Scaling because, for example, observers do not perceive inner corners as being further away than outer corners when the stimulus is viewed as a three dimensional model (McGraw and Stanford, 1994; Stacey and Pike, 1970), a somewhat attenuated version of the illusion can occur when dots are employed instead of lines (GreistBousquet and Schiffman, 1981; Fig. 1B), when overt three dimensional cues are provided (DeLucia and Hochberg, 1991), or even when the stimuli are examined haptically. Despite this, Gregory's idea continues to enjoy popularity in current textbooks on the topic (Goldstein, 2009), likely due to several reasons. First, it is fairly well established that perceived distance can influence the perceived size of an object, such a mechanism being necessary for size constancy to occur (Holway and Boring, 1941). Second, the examples held up as evidence against Gregory's mechanism fail to rule out Misapplied Size Constancy Scaling directly for the original illusion, instead assuming that because the illusion persists in the absence of depth cues (e.g., the dot version), depth cue information is not playing a role in the illusion at all. 
For example, there is evidence that the haptic version of Muller-Lyer is governed by different mechanisms than those found in the traditional visual version. Specifically, when cross-modal versions of Muller-Lyer are created (in which the stimuli are partly haptic and partly visual), the illusion size is greatly diminished, implying different mechanisms may be responsible for the haptic and visual versions of the illusion. Put more broadly, it is possible that more than one mechanism may be playing a role in the illusion depending on the type of stimulus employed and a misapplied constancy scaling mechanism might be one of them. In fact, in favor of this last possibility is recent neurological evidence supportive of a misapplied size constancy scaling account for the visual version of the MullerLyer (Weidner and Fink, 2007).

Day (1989) 'Conflicting Cues' explanation states that the illusion is a consequence of the overall lengths of the two component stimuli being different. Specifically, the arrowheads themselves contribute to the overall perception of length, resulting in an averaging process, where the perceived length of the line is a function of the actual length integrated with the length of the entire stimulus. In other words, the line bound by inward-pointing arrowheads is seen as longer, due to the additional length of the arrowheads themselves contributing to that length estimate.

The Confusion Hypothesis (Sekuler and Erlebacher, 1971) is somewhat similar to Conflicting Cues, in that according to this explanation the length of the line is influenced by the inter-tip distances between the bounding arrowheads. So, the inward-pointing arrowheads have large inter-tip distances that "stretch" the perceived line length and the outward-pointing arrowheads have small inter-tip distances, compressing the perceived line length. This is not the only such explanation put forth for Muller-Lyer that implies a perceptual 'stretching' of the fins-in and 'compression' of the fins-out (for example, Bulatov et al. (1997) spatial filter model, or Findlay (1982) 'center of gravity' for alternatives). The work presented here is not intended to specifically test between these various alternatives, since for the most part, they will make similar predictions for the stimulus configurations employed. For the purpose of brevity, however, Confusion Hypothesis will be used to denote this class of explanation.

This study was designed to test between competing predictions made by these three perspectives by systematically varying the size or orientation of two variants of the classic MullerLyer: the 'dumbbell' (Parker and Newbigging, 1963) version, where the bounding elements are circles (Fig. 1C) and the 'spectacle' version, where the bounding elements are ovals (Fig. 1D).

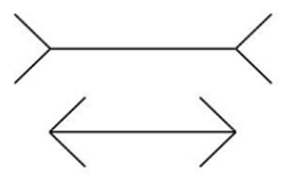

Traditional version

(a)

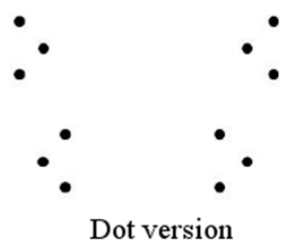

(b)

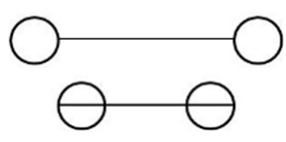

Dumbbell version

(c)

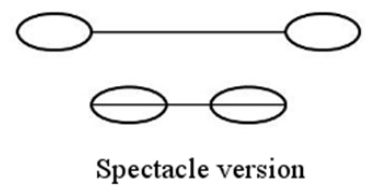

(d)

Fig. 1: Various configurations of the Muller-Lyer illusion

Each of the previously-mentioned explanations makes a different set of predictions of change in illusion strength as the bounding elements are changed in terms of either their size (in the case of dumbbell stimuli) or orientation (in the case of spectacle stimuli). What follows is a more detailed explanation of these.

Although critics point out that there are no apparent depth cues for the dumbbell stimuli (hence no associated distance inference mechanism should be engaged), it is possible to salvage a size constancy scaling explanation for this illusion if the circles were perceived as three-dimensional dumbbells/bowling balls and the line perceived as a physical bar. If that is the case, then the line that overlaps the circles would be perceived as 'on top' of the bowling balls and therefore closer to the observer, than the bar lying between the bowling balls. In this way, misapplied size constancy scaling might still be active, since the line on top of the bounding elements, if seen by the perceptual system as closer, should be perceived to be smaller than the line lying between the bounding elements. Note that this 


\begin{tabular}{|c|c|c|c|c|c|}
\hline \multicolumn{6}{|c|}{$(\uparrow=$ Relative increase $\downarrow$ = Relative decrease.$-=$ No change) } \\
\hline & Comparison & $\underline{\mathrm{MSCS}}$ & $\underline{\mathrm{CC}}$ & $\underline{\mathrm{CH}}$ & obtained \\
\hline a) & $\because$ vs $\because$ & 一 & $\uparrow$ & $\uparrow$ & $\downarrow$ \\
\hline b) & $\because$ vs $\because$ & $\uparrow$ & 一 & $\uparrow$ & $\uparrow$ \\
\hline c) & $\because$ vs $\stackrel{\circ}{\circ}$ & $\uparrow$ & $\uparrow$ & $\uparrow$ & $\uparrow$ \\
\hline d) & $\rightarrow 0$ vs $\because 0$ & - & $\uparrow$ & $\uparrow$ & $\uparrow$ \\
\hline e) & $a_{0 \rightarrow 0}$ vs $\longrightarrow$ & - & 一 & $\uparrow$ & $\uparrow$ \\
\hline f) & $\because$ vs $\because$ & - & $\uparrow$ & $\uparrow$ & $\uparrow$ \\
\hline
\end{tabular}

Fig. 2: Predicted and obtained changes in illusion strength as a result of change in bounding element size or orientation (MSCS = Misapplied Size Constancy Scaling; $\mathrm{CC}=$ Conflicting Cues hypothesis; $\mathrm{CH}=$ Confusion Hypothesis)

prediction remains the same whether or not the larger circles are taken as larger bowling balls, or are perceived as being the same size but closer to the observer.

The prediction that follows from this according to Misapplied Size Constancy Scaling is that increasing the size of the inner bounding circles should result in an increase in the size misperception of the lines (due to it now being perceived as closer than if small circles were employed), while increasing the outer bounding circles should not (since, in either case, the line is lying between the bounding elements). Conversely, when spectacle bounding elements are employed, where the orientation of the oval is varied for inner and outer bounding elements, Misapplied Size Constancy Scaling would predict no effect on illusion strength due to the fact that rotating the ovals would not produce a change in perceived distance, hence the illusion strengths should be constant across all spectacle trial types.

According to the Conflicting Cues account, increasing the size of the outer bounding circles should increase the strength of the illusion (the resulting stimulus would now be longer overall), whereas, with no new length being added to the stimulus, increasing the size of the inner circles should not significantly affect the illusion. Also, Conflicting Cues would predict that when spectacle bounding elements are employed, then rotating the outer, but not the inner, bounding ovals should result in increases in the illusion strength.
Finally, the Confusion Hypothesis would predict that increasing either the inner or outer circles of the dumbbell versions, or rotating either the inner or outer ovals of the spectacle stimuli, should result in increases in illusion strength relative to baseline, since in all cases, the intertype distances of the bounding elements would be different.

In summary, all three models vary in terms of the pattern of outcome that would arise when the bounding elements are varied. A summary of these predictions, as well as a summary of the obtained outcomes, can be found in Fig. 2.

\section{MATERIALS AND METHODS}

Participants: About 50 male and female Psychology undergraduates participated in exchange for partial course credit. The median age for the sample was 19 years old. All participants had normal or corrected-tonormal eyesight.

Stimuli: All stimuli consisted of either a spectacle or dumbbell version of Muller-Lyer, oriented horizontally and appearing in random places on the screen on each trial. The two component figures for each instance of the illusion were never aligned vertically.

For the dumbbell stimuli, the small and large circles were one and two $\mathrm{cm}$ in diameter, respectively. By systematically varying the size of the bounding circles to be either one (small) or two (large) centimetres in diameter, the four configurations of the illusion were constructed (small outer/small inner, small outer/large inner, large outer/small inner and large outer/large inner).

For the spectacle stimuli, the oval measured $1 \mathrm{~cm}$ in width and $2 \mathrm{~cm}$ in length. Similar configurations to those seen in the dumbbell configurations were employed by orienting the bounding ovals either vertically (corresponding to 'small' according to Conflicting Cues or Confusion Hypothesis) or horizontally (corresponding to 'large' according to Conflicting Cues or Confusion Hypothesis). Examples of all eight stimulus types can be seen in Fig. 3. The standard stimulus (always the lower of the two in the display) was set at $9 \mathrm{~cm}$ in length. The comparison figure (that participants could adjust) started out either $4 \mathrm{~cm}$ longer or $4 \mathrm{~cm}$ shorter than the standard, giving rise to two adjustments occurring for each stimulus configuration.

Procedure: The method of adjustment was employed, with participants controlling the length of the upper- 
most line. Participants sat approximately $57 \mathrm{~cm}$ away from the Samsung SyncMaster 920BM flat-screen monitor $\left(1 \mathrm{~cm}=1^{\circ}\right.$ visual angle $)$ and were instructed not to move forward or back through the course of the study, which took approximately ten minutes to complete. Each participant received a random ordering of the stimuli for adjustment.

\section{RESULTS}

For each trial, the difference (in $\mathrm{cm}$ ) between the upper and lower line following adjustment for each trial type and participant was computed as the measure of illusion strength (lower line length-upper line length). From these, mean PSEs consisting of the average of the two adjustments for a stimulus type were computed and formed the "mean illusion size" for the respective stimulus type used in subsequent analyses. Figure 3 displays the obtained mean illusion strengths and standard errors for each stimulus type.

Based on the predicted outcomes depicted in Fig. 2, a set of six planned comparisons was carried out using a Bonferroni adjustment (each with a two-tailed criterion of $\mathrm{p}<0.008$ ), comparing the illusion size obtained for each 'baseline' stimulus to each of the other respective configurations for that stimulus type. Table 1 shows the outcome of these tests for each of the comparisons. In summary, all six obtained differences were significant at $\mathrm{p}<0.005$. As can be seen from the last column in Fig. 2, relative to the baseline configuration, all but one variation on bounding element size or orientation gave rise to significant increases in illusion strength.

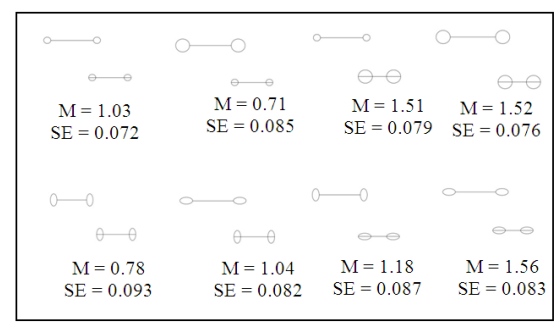

Fig. 3: Illusion strength Means and Standard Errors for each stimulus type

Table 1: Pairwise comparisons for each of the six contrasts found in Fig. 2

\begin{tabular}{lcc}
\hline Comparisons from Fig. 2 & $\mathrm{t}$ & Sig. (2-tailed) \\
\hline a) & -3.281 & 0.002 \\
b) & 6.505 & $<0.001$ \\
c) & 5.775 & $<0.001$ \\
d) & 3.036 & 0.004 \\
e) & 4.134 & $<0.001$ \\
f) & 7.749 & $<0.001$ \\
\hline
\end{tabular}

The sole exception, in which the outer bounding elements for the dumbbell stimuli were increased in size, resulted in an unexpected significant decrease in illusion strength.

\section{DISCUSSION}

None of the models by themselves is able to fully account for the results obtained in this study. First, for the most part, the data were not supportive of Misapplied Size Constancy Scaling. Re-orienting the bounding ovals of the spectacle stimuli should not have given rise to any change in perceived distance from the observer and Misapplied Size Constancy Scaling correspondingly would predict that this manipulation should not result in any change in illusion strengths. Second, Conflicting Cues theory failed to predict the robust increase in illusion strength when the inner bounding elements of the dumbbell configuration were increased in size. Finally, although the predictions made by Confusion Hypothesis conformed most closely to the obtained pattern of change in illusion strengths, it was still the case that increasing the size of the outer dumbbell elements gave rise to a significant decrease in illusion strength, rather than the predicted increase. In fact, this anomalous result would not be predicted by any account for Muller-Lyer that is based on the outward inducing elements contributing to an overestimate of the line length, such as Ginsberg (1986) spatial filter theory, Festinger et al. (1968) visual scanning-time hypothesis, or Gilster and KuhtzBuschbeck (2010) center of gravity notion.

It would appear, then, that the pattern of results is due to a combination of several mechanisms operating simultaneously, that may have varied between spectacle and dumbbell configurations. First, for the dumbbell stimuli, the act of increasing the bounding elements may have resulted in those circles no longer being perceived as "part of" the entire stimulus and instead were seen as separate entities to the line. Such effects have been previously reported (Fellows, 1967; Restle and Decker, 1977), however the size of the bounding elements required for such an effect to occur usually extend well beyond those used here. It is possible, though, that the perceptual detachment point is less extreme when circles are used as bounding elements as opposed to when the more common fin configurations are employed. This would result in Confusion Hypothesis predicting a slight drop in illusion strength relative to the baseline for either the outer or inner (or both) bounding elements were increased for the dumbbell stimuli, because the larger circles would no longer be perceptually integrated with the lines. 
Unfortunately, though there was a significant drop in illusion strength when increasing the size of the outer dumbbells, there was a corresponding significant increase in illusion strength when the size of the inner bounding dumbbells were increased, contrary to Confusion Hypothesis' modified prediction. In other words, if this perceptual segregation of shaft and bounding elements for the dumbbell stimuli is taking place, there must be some other mechanism playing a role besides that specified by Confusion Hypothesis or Conflicting Cues, to fully account for the obtained pattern.

\section{CONCLUSION}

Interestingly, this perceptual detachment of bounding elements from the line would still allow Misapplied Size Constancy Scaling to function, because the distance cues provided by those elements do not require they be perceived as being part of a single integrated whole stimulus. Additionally, decreases in illusion strength when the outer bounding dumbbells increased might be accounted for by something akin to the Ebbinghaus illusion (Roberts et al., 2005) such that the larger bounding elements might perceptually shrink the size of the line they bound (Nemati, 2009). Note, however, that an explanation based solely on the Ebbinghaus illusion cannot entirely account for the obtained data from the dumbbell stimuli, since it does not offer any explanation for the illusion occurring in the baseline condition, nor does it predict that the illusion size should be different when both inner and outer bounding elements are increased. In other words, for the dumbbell stimuli, Misapplied Size Constancy Scaling and the Ebbinghaus illusion combined could account for the obtained pattern of results. Such a possibility is not so far-fetched, given that there is no reason to rule out the possibility that more than one mechanism may be contributing to the illusion.

The spectacle stimuli did not activate any overt depth cues, so Misapplied Size Constancy Scaling would not predict any change in illusion strength, however the 'thinner' bounding spectacles when made "large" (when the oval was horizontal) may still have been perceptually integrated with the line, allowing the sort of mechanism proposed by Confusion Hypothesis to remain active, resulting in the pattern of change obtained.

Given the difficulty researchers have had trying to develop a single model to fully account for the illusion in all its incarnations, it appears likely that more than one mechanism is responsible for it and what mechanisms are operating depend to a great extent on the specific configuration (haptic, dot, traditional) of Muller-Lyer employed in the study. If dot or haptic stimuli are employed, it rules out a Misapplied Size Constancy Scaling mechanism operating to produce illusions for those, but not necessarily all, versions of Muller Lyer. Likewise, the existence of the illusion under such a wide variety of conditions virtually precludes the notion of a single mechanism governing all of them, given the wide range of stimuli and sensory modalities within which it appears. Researchers, therefore, might be wise to entertain the possibility that there are multiple means of producing what appears on the surface to be a single illusion, instead of continuing to pursue a Grand Unifying Theory for Muller-Lyer in all its various disguises.

\section{REFERENCES}

Bulatov, A., A. Bertulis and L. Mickiene, 1997. Geometrical illusions: Study and modelling. Biol. Cyber, 77: 395-406. PMID: 9433754

Day, R.H., 1989. Natural and artificial cues, perceptual compromise and the basis of veridical and illusory perception. In: Human Information Processing: Measures,Mechanisms and Models. Vickers, D. and P.L. Smith (Eds.). North Holland, California, pp: 107-129, ISBN-10: 044488520X

Delucia, P.R. and J. Hochberg, 1991. Geometrical illusions in solid objects under ordinary viewing conditions. Attention Perception Psychophys., 50: 547-554. DOI: 10.3758/BF03207539

Fellows, B.J., 1967. Reversal of the Muller-Lyer illusion with changes in the length of the inter-fins line. Q. J. Exp. Psychol., 19: 208-214. DOI: 10.1080/14640746708400094

Festinger, L., C.W. White and M.R. Allyn, 1968. Eye movements and decrement in the Muller-Lyer illusion. Perception Psychophys., 3: 376-382.

Findlay, J.M., 1982. Global visual processing for saccadic eye movements. Vision Res., 22: 1033-1045. DOI: 10.1016/0042-6989(82)90040-2

Gilster, R. and J.P. Kuhtz-Buschbeck., 2010. The Muller-Lyer illusion: Investigation of a center of gravity effect on the amplitudes of saccades. J. Vision, 10: 1-13. DOI: 10.1167/10.1.11

Ginsberg, A.P., 1986. Spatial Filtering and Visual form Perception. In: Handbook of Perception and Human Performance, Boff, K.R., L. Kaufman and J.P. Thomas (Eds.). Wiley, New York, ISBN-10: 0471850616, pp: 34-41.

Goldstein, E.B., 2009. Sensation and Perception. 8th Edn., Wadsworth Publishing, USA., ISBN-10: 0495601497, pp: 459. 
Gregory, R.L., 1997. Eye and Brain: The Psychology of Seeing. 5th Edn., Princeton University Press, USA., ISBN-10: 0691048371, pp: 277.

Greist-Bousquet, S. and H.R. Schiffman., 1981. The many illusions of the Muller-Lyer: Comparisons of the wings-in and wings-out illusions and manipulations of standard and dot forms. Perception,10: 147-154. DOI: 10.1068/p100147

Holway, A.H. and E.G. Boring, 1941. Determinants of apparent visual size with distance variant. Am. J. Psychol., 54: 21-37.

Madden, T.M. and G.S. Burt, 1981. Inappropriate constancy scaling theory and the Mueller-Lyer illusion.Percep. Mot. Skills, 52: 211-218. PMID: 7232084

Nemati, F., 2009. Size and direction of distortion in geometric-optical illusions: Conciliation between the Müller-Lyer and Titchener configurations. Perception, 38: 1585-1600. PMID: 20120258

Parker, N.I. and P.L. Newbigging, 1963. Magnitude and decrement of the Muller-Lyer illusion as a function of pre-training. Can. J. Psychol., 17: 134-140. PMID: 13941492
Restle, F. And J. Decker, 1977. Size of the MuellerLyer illusion as a function of its dimensions: Theory and data. Attention Perception Psychophys., 21: 489-503. DOI: 10.3758/BF03198729

Roberts, B., M.G. Harris and T.A. Yates, 2005. The roles of inducer size and distance in the Ebbinghaus illusion (Titchener circles). Perception, 34: 847-856. PMID: 16124270

Robinson, J.O., 1998. The Psychology of Visual Illusion. 1st Edn., Dover Publishing, Canada, ISBN-10: 0486404498, pp: 288.

Rock, I., 1975. An Introduction to Perception. 1st Edn., Macmillan, New York, ISBN-10: 0024024902, pp: 580.

Sekuler, R. and A. Erlebacher, 1971. The two illusions of Muller-Lyer: Confusion theory reexamined. Am. J. Psychol., 84: 477-486.

Weidner, R. and G.R. Fink, 2007. The neural mechanisms underlying the Muller-Lyer illusion and its interaction with visuospatial judgments. Cerebral Cortex, 17: 878-884. DOI: 10.1093/cercor/bhk042 